\title{
Usos y significados de la noción de naturaleza en el modelo de "parto y crianza fisiológica" en la ciudad de Buenos Aires
}

\section{María Jimena Mantilla, ${ }^{1,2}$}

\author{
1 Instituto de Investigaciones Gino Germani, Universidad de Buenos Aires, Buenos Aires, Argentina. \\ 2 Consejo Nacional de Investigaciones Científicas y Técnicas, Buenos Aires, Argentina. \\ ORCiD: 0000-0003-4206-0952 \\ Correo electrónico: mantillamariajimena@gmail.com
}

Recibido:

diciembre de 2018

Aceptado:

agosto de 2019

doi: 10.34096/runa.v41i1.6596

\section{Resumen}

El objetivo de este artículo es analizar desde una perspectiva sociológica cómo los discursos de parto fisiológico y crianza fisiológica conciben y dan sentido a la noción de "naturaleza". A partir de una investigación cualitativa de tipo etnográfico sugiero que el conocimiento de la fisiología tiene la potencialidad de constituirse en una retórica de empoderamiento de la mujeres a partir de generarles confianza en su capacidad de parir y seguridad en los procesos naturales y espontáneos de sus cuerpos, al tiempo que el conocimiento de la fisiología redefine los modos de escuchar, atender y acompañar procesos vitales de la infancia de sus hijos.

Uses and meanings of the notion of nature in the model of "childbirth and physiological upbringing" in the city of Buenos Aires

\begin{abstract}
The aim of this article is to analyze from a sociological perspective how discourses of physiological labor and upbringing conceive the notion of "nature". Based on an ethnographic research, I suggest that knowledge of physiology has the potential to become a rhetoric of women's empowerment by generating confidence in their ability to give birth and security in the natural and spontaneous processes of their bodies. The knowledge of physiology also redefines the ways of listening, attending and accompanying the vital processes of their children's childhood.
\end{abstract}

Palavras-chave

Fisiología; Parto; Crianza; Empoderamiento; Naturaleza

\section{Palabras clave}

Phisiology; Labor; Upbringing; Empowerment; Nature 


\section{Usos e significados da noção de natureza no modelo de "parto e educação fisiológica" na cidade de Buenos Aires}

\section{Resumo}

Palavras-chave

Fisiologia; Parto; Parentalidade; Empoderamento; Natureza

1. Se utiliza a lo largo del texto el entrecomillado para identificar las categorías nativas.

2. El término "cría” se utiliza frecuentemente en este enfoque, en especial cuando se hace referencia a las etapas de nacimiento y primer año del/a niño/a. Mediante esta palabra se refuerza la asociación entre los humanos y los demás mamíferos, entendiendo por ello que bebés y niños/as pequeños/ as necesitan -para su consecuente desarrollo y maduración biológica y psicológica- un proceso de contacto corporal intensivo por parte de su cuidador principal, preferentemente su madre.
O objetivo deste artigo é analisar, a partir de uma perspectiva sociológica, como o parto fisiológico e a educação fisiológica concebm e dão sentido à noção da "natureza". Com base na pesquisa etnográfica qualitativa, sugiro que o conhecimento da fisiologia tem o potencial de se tornar uma retórica do empoderamento das mulheres a partir de construir a confiança em sua capacidade de dar à luz e segurança nas processos naturais e espontâneos de seus corpos. Ao mesmo tempo, o conhecimento da fisiologia redefine formas de escutar, cuidar e acompanhar os processos vitais da infância de seus filhos.

\section{Introducción}

El objetivo de este artículo es analizar desde una perspectiva sociológica cómo los discursos de "parto fisiológico"1 y "crianza fisiológica" conciben y dan sentido a la noción de "naturaleza", categoría movilizada en dichos discursos.

En las últimas décadas comenzó a adquirir mayor visibilidad un estilo de concebir la maternidad denominado "natural", centrado en la figura y cuidado del/a niña/o, que se caracteriza por una mayor presencia de ambos progenitores en la etapa de la primera infancia de sus hijos/as y el respeto por sus necesidades evolutivas y emocionales. Diversos autores documentaron el fenómeno en otras partes del mundo (Tornquist, 2002, 2004; Fedele 2016) y recientemente en Argentina (Abdala, 2016; Mantilla, 2019). Esta nueva tendencia va de la mano del cuestionamiento a modelos tradicionales de crianza y de la organización doméstica del cuidado infantil al tiempo que confluye con el movimiento de transformación de la hipermedicalización del parto, y con los activismos en contra de la "violencia obstétrica" y a favor del respeto por la "fisiología del parto y el nacimiento".

En esa línea, los términos "crianza respetuosa", "parto natural", "parto respetado", "madres naturales" y "crianza fisiológica", si bien aluden a cuestiones diferentes, resuenan en un reportorio afín de prácticas cuya principal característica en común es la búsqueda y la elección de una gestación, parto, nacimiento y crianza en mayor conexión con la naturaleza y los ritmos naturales del cuerpo de las mujeres y sus "crías". ${ }^{2}$ La naturaleza, aquí, es interpretada bajo la figura de la fisiología y ello implica el seguimiento de los procesos naturales y espontáneos del cuerpo. Las mujeres en conexión con sus procesos fisiológicos y el de sus hijos/as acceden a un conocimiento efectivo de cómo parir y criar.

Según la historiadora Martucci (2015), la "maternidad natural" descansa en un entendimiento científico de la naturaleza y la maternidad en el cual los procesos fisiológicos y emocionales están conectados. La autora afirma que este enfoque emerge en tensión con la ideología de la maternidad científica, 
paradigma dominante del siglo $\mathrm{XX}$, que se basa en la autoridad descorporizada y masculina y la mediación tecnológica de expertos en los procesos maternales, que incluyen el nacimiento hospitalario altamente intervenido y la alimentación infantil con leche de fórmula. A ese estilo cultural de maternidad se opone radicalmente la "maternidad natural" aquí analizada.

En Argentina, la creciente aparición de espacios y grupos presenciales y/o virtuales que convocan a compartir experiencias de parto y crianza y difunden información alineada con este enfoque da cuenta de la magnitud de este fenómeno cultural. El parto natural y la crianza natural se han instalado también en los medios de comunicación mediante los testimonios de actrices y modelos que se definen como practicantes y defensoras de este modelo (Sánchez de Bustamente, 2017). Sin duda, el cruce entre la elección personal de cómo parir y criar y la defensa pública de estas prácticas es un rasgo significativo en las trayectorias de mujeres interpeladas por el modelo de "crianza natural". Pero ¿qué significa la naturaleza en este marco? ¿Qué nociones al respecto circulan en estos espacios y cuáles son los sentidos que adquieren en las prácticas de las mujeres? ¿Con qué otras nociones se asocia? ¿Y a cuáles otras se opone, por ejemplo, peligro e imprevisibilidad, cómo la suponen los médicos?

La investigación se inscribe en la línea de estudios socioculturales sobre parto, maternidad y crianza (Carli, 1999; Martin, 2006; Cosse, 2009, 2010a y b; Schwarz, 2010; Felitti, 2012; Castrillo, 2014; Faur, 2014, entre otros). Si bien aún las investigaciones sobre la temática del parto y la crianza naturales son escasas, los trabajos de Bobel (2002) en Estados Unidos y su análisis de las "maternidades naturales"; los de Faircloth (2013) sobre la crianza con apego y la defensa del amamantamiento en el Reino Unido; los de Fedele (2016) sobre las maternidades holísticas en Portugal; y Tornquist $(2002,2004)$ con su etnografía acerca del ideario y las prácticas del movimiento por la humanización del parto en Brasil, problematizan las experiencias de mujeres de clases medias que adscriben a estos enfoques. En el plano local son antecedentes relevantes el trabajo de Abdala (2016) sobre las experiencias de gestación y maternidad de mujeres interpeladas por la búsqueda del parto y la crianza natural en la ciudad de Santa Fe, y las investigaciones sobre violencia obstétrica de Fornes (2011), las estrategias de activismo en parto respetado (Jerez, 2015), y las experiencias de preparación para un parto respetado en La Plata (Castrillo, 2014). Estos estudios configuran el espacio de discusión en el que se inscribe este trabajo.

Desde la perspectiva de algunos estudios feministas (Badinter, 1981, 2010; Del Olmo, 2013; Meruane, 2018; Tenenbaum, 2019, entre otros) y también desde la experiencia de algunas mujeres, la perspectiva fisiológica es susceptible de tornarse en un conjunto de ideales normativos y dogmáticos en torno a la maternidad que poco tienen que ver con un ejercicio genuino de las elecciones, el deseo y la autonomía de las mujeres. No obstante, y en consonancia con los hallazgos de esta investigación, sugiero que el conocimiento de la "fisiología" tiene la potencialidad de constituirse en una retórica de empoderamiento ${ }^{3}$ de las mujeres a partir de generarles confianza en su capacidad de parir y seguridad en los procesos "naturales" y espontáneos de sus cuerpos, al tiempo que redefine los modos de escuchar, atender y acompañar procesos vitales de la infancia de sus hijos/as. Es evidente que esta dimensión de empoderamiento se tensiona con las posibilidades efectivas de las mujeres, sus propios deseos y los condicionantes que estructuran sus trayectorias vitales; pero nada de ello anula su potencia.
3. La noción de empoderamiento tiene múltiples usos y ligazones a diferentes perspectivas; en este caso, recupero el sentido en el que la usa Susana Tornquist (2004) para analizar el ideario de humanización del parto, que refiere a cómo las mujeres, gracias a este tipo de retórica que naturaliza el proceso de dar a luz, recuperan su propia capacidad de parir y, en este sentido, se vuelven protagonistas de sus partos. La noción de empoderamiento y sus modos de operar en las vivencias de las mujeres constituyen un tópico que se explorará en otros trabajos. 
El artículo se organiza de la siguiente forma: en primer lugar, describo sucintamente los elementos que definen a la perspectiva "fisiológica"; en segundo lugar, me detengo en analizar cómo opera en la construcción de significados durante la experiencia del parto y el nacimiento; y finalmente, analizo cómo la fisiología se inscribe en el lenguaje de las hormonas, los procesos biológicos y los tiempos propios de la naturaleza de los cuerpos de las mujeres y sus bebés y se transforma así en una retórica que provee a las mujeres agencia y autonomía en las escenas sociales del parto y la crianza.

\section{Notas metodológicas}

Este texto forma parte de una investigación en curso en la que analizo desde una perspectiva sociológica las experiencias y trayectorias de mujeres interpeladas por el modelo de "parto natural" y "crianza natural" en la Ciudad Autónoma de Buenos Aires y el Gran Buenos Aires. La estrategia metodológica que guía la investigación consiste en un diseño cualitativo de tipo etnográfico en base a fuentes primarias (entrevistas en profundidad y observaciones participantes) y secundarias (documentos, revistas, documentales y libros relativos a la temática).

Si bien el trabajo de campo está en proceso, hasta la fecha se realizaron 27 entrevistas en profundidad (de dos horas promedio de duración, y en ocasiones en más de una sesión) con mujeres madres de sectores medios de la ciudad de Buenos Aires y el Gran Buenos Aires que siguen este tipo de crianza. Algunas también se dedican a la difusión de estas ideas, ya sea mediante el activismo y la participación pública, ya sea mediante su recorrido laboral desarrollando un rol profesional vinculado con el mundo de la "crianza respetuosa" (doulas, puericulturas, asesoras de crianza, coordinadoras de grupos de crianza, entre otras formas de expertise caras a este modelo).

Además de las entrevistas, participé de 19 reuniones vinculadas a la difusión de este modelo: cursos de preparación para el parto; charlas donde se brinda información sobre parto respetado, parto después de cesárea; jornadas de crianza respetuosa donde se ofrecen charlas, talleres y venta de accesorios vinculados (fulares, portabebés, pañales de tela, etc.); presentaciones de agrupaciones feministas que abordan la problemática de la violencia obstétrica; talleres de alimentación respetuosa en los que se acompaña a las familias en el ingreso de la alimentación haciendo hincapié en que sea el bebé quien gestione los alimentos por sí mismo; y talleres de límites y acompañamiento emocional de la crianza. Asimismo, como parte del trabajo de campo, documenté intercambios y participé de grupos de crianza y de parto respetado en las redes sociales.

Por último, la sensibilidad con estas temáticas se inscribe en mi propia llegada a la maternidad. Ya durante el embarazo, la espera de mi hija se transformó, entre otras cosas, en una búsqueda activa de información acerca del proceso de parir y nacer. Recuerdo mi sorpresa y preocupación al leer un relato en un foro de maternidad, que mencionaba cómo a un bebé recién nacido le habían quebrado la clavícula en el "expulsivo". Comprendí que no podía dejar en manos de los médicos la llegada al mundo de Catalina y desde entonces comencé a toparme con las palabras de "parto humanizado", "parto respetado" y "violencia obstétrica", entre otras. Una vez convertida en madre, en sintonía con mis propias experiencias, empecé a prestar atención a otras palabras que circulaban por las redes sociales: la "crianza con apego", el "colecho", la "lactancia prolongada" y el "destete fisiológico". En medio de todo ese abanico de 
temáticas, la pregunta por el significado de la noción de naturaleza comenzó a ser un elemento central. Pues todas estas miradas sobre la maternidad y la paternidad, los nacimientos y los niños tienen en común una idea acerca de qué es y cómo funciona la naturaleza y sobre cómo seguir el camino que esta indica. Apelan a lo natural como marca distintiva, en oposición a otros modelos de maternidad, nacimiento y crianza. "Seguir la naturaleza" se transformó, en mi caso, en una experiencia y guía, a veces opaca y errática, y siempre cultural e interpretativa para la maternidad. Me convertí en una mamá que da la teta a una niña "grande" desde la mirada social hegemónica, y con ello aprendí las luces y sombras del dar del propio cuerpo a contrapelo de la norma social.

\section{La fisiología en el parto y la crianza: primeras aproximaciones}

La perspectiva del "parto fisiológico" y la "crianza fisiológica" se centra en el respeto por los procesos fisiológicos del parto y el nacimiento, los tiempos madurativos de los/as niños/as y la consideración de sus necesidades de apego seguro brindadas por un entorno familiar adecuado a estos fines. La crianza con apego (attachment parenting), planteada por William y Martha Sears (1960) a partir de la teoría del apego de Bowlby, sustenta este enfoque. Este estilo de crianza se vincula también al "continuum", un concepto clave para la comprensión de la crianza respetuosa, formulado por Jean Liedloff en los años setenta y al que define como:

\section{la secuencia de experiencias que corresponde a las expectativas y tendencias de nuestra especie en un entorno consecuente con aquello en lo que esas expectativas $y$ tendencias se formaron. Incluye que las otras personas que forman parte de aquel entorno se comporten y nos traten adecuadamente. (Liedloff, 2011, p. 54)}

Desde la fisiología se entiende que el desarrollo de niños y niñas es espontáneo y ocurre en los tiempos propios y únicos de cada niño/a (Mantilla, 2019). En este sentido, la fisiología articula una dimensión universal con una individual en tanto las etapas, tanto del parto como del desarrollo infantil, son similares para todos los seres vivos pero se manifiestan con características propias y ritmos singulares. Tal cómo me contaba Adriana, ${ }^{4}$ una de las especialistas en desarrollo infantil que mayor recorrido y relevancia pública tienen en Argentina: "Todos los bebés de todo el mundo nacemos con la misma expectativa, que es encontrarnos con el organismo que nos dio la vida y que es nuestra madre" (entrevista con especialista en desarrollo infantil y crianza fisiológica, 12 de abril de 2018). Desde esta mirada, las expectativas de ser amamantados, cobijados y acompañados en los procesos de desarrollo son iguales para todas las niñas y niños y no están sujetas a la interpretación cultural, porque las necesidades de los bebés entran en un orden precultural. La cultura, en todo caso, es la organización social de una respuesta a esa necesidad. En consecuencia, lo que aquí se promueve es una respuesta cultural lo más cercana al respeto por esa fisiología que se postula como inmanente.

En esta visión, la función del adulto es vivenciar y acompañar los procesos fisiológicos de la gestación, el nacimiento y la infancia: el ritmo del sueño, las necesidades de amamantamiento, alimentación, colecho, porteo y control de esfínteres son etapas ligadas a las expectativas fisiológicas de los niños y las niñas. Es fundamental el respeto de los tiempos propios y naturales de ellos y ellas, sin imponer desde afuera ritmos externos pensados por los adultos. El desafío, para estos padres y madres, no está en imponer su autoridad como en el modelo tradicional de crianza, sino en entender, acompañar y respetar
4. Los nombres de instituciones y personas citados son ficticios, respetando las normas de anonimato y confidencialidad propias de la investigación cualitativa. 
5. La Organización Mundial de la Salud (2015) alertó recientemente sobre una "epidemia de cesáreas" a nivel mundial, y colocó a Argentina entre los países que tienen una de las mayores tasas y que además duplica lo indicado por la OMS. Mientras éste sostiene que no existe ninguna evidencia científica para que las cesáreas representen más del $15 \%$ de los partos, en Argentina esa tasa es del $30 \%$, que, según las estimaciones, en el sector privado puede alcanzar alrededor del $65 \%$. Más allá del número alarmante de cesáreas, no hay datos precisos sobre intervenciones innecesarias en los partos vaginales como el uso de oxitocina sintética, la rotura de bolsa, entre otras.

6. La figura de la doula emerge con fuerza en Argentina en los últimos años de la mano de la concientización progresiva sobre la importancia de reconectar a las mujeres con sus capacidades de parir. Se trata de mujeres que se dedican a acompañar en las etapas de gestación, parto y posparto. El rol consiste en brindar información sobre la legislación, los derechos que asisten a las parturientas y sus bebés, y estar a su disposición mediante la escucha, la presencia y la contención durante la gestación y el parto. esas necesidades fisiológicas mediante la observación y el acompañamiento. La misma posición cabe frente a los procesos de gestación y parto: lo que se espera es acompañar la fisiología, y ello implica favorecer la ocurrencia espontánea del parir y nacer en los tiempos singulares de cada díada sin intervenir en ese proceso.

Teniendo en cuenta esta breve introducción a los lineamientos generales de la perspectiva fisiológica, en el próximo apartado me centro en describir y analizar sus efectos en la construcción de una retórica del parto.

\section{La retórica fisiológica en la experiencia del parto}

La fisiología del parto y nacimiento, y la importancia de la experiencia fisiológica para las mujeres y los recién nacidos forman parte de la retórica de valoración del parir sin intervenciones médicas en los casos de gestaciones saludables. Por ello, en las charlas y talleres de parto fisiológico es frecuente que se difunda información acerca de los mecanismos biológicos que están en juego en estos procesos vitales y cómo estos afectan tanto a los bebés en sus procesos de desarrollo como a las mujeres en la constitución de prácticas de cuidado y sentimientos de apego hacia sus "crías".

En primer lugar, desde la perspectiva fisiológica, el parto se define como un proceso involuntario, y el proceso fisiológico que se desencadena en el acto de parir, como una capacidad inherente a la condición femenina. Al colocar el parto en el ámbito de la naturaleza femenina, se invierte la retórica cultural hegemónica sobre la gestación y el parto que los define como eventos médicos, primordialmente por fuera del control y gestión de las mujeres gestantes. La apelación a una temporalidad natural del proceso de parir, que es a su vez individual y propia de cada mujer, se opone al modo estandarizado en el que se entiende la temporalidad desde el enfoque biomédico. Se abre un espacio de incertidumbre e indeterminación que es clave, como veremos más adelante, para comprender el transcurso efectivo de un parto sin intervenciones médicas innecesarias.

La definición de parto fisiológico se difunde y se promociona en cada encuentro y espacio de difusión de este enfoque. Las charlas, círculos y talleres insisten sobre este punto pues, si bien es exactamente la información que promueve la Organización Mundial de la Salud hace tres décadas respecto de la atención del parto, existe un notable desfasaje con las prácticas clínicas en Argentina de buena parte de la obstetricia, definidas por su elevado nivel de intervencionismo. ${ }^{5}$ En uno de los encuentros de "Gestar", un espacio para futuros padres y madres que se centra en transmitir información fisiológica del embarazo, el parto, la lactancia y el puerperio, Juana, una de las doulas ${ }^{6}$ coordinadoras, abrió la reunión con la siguiente pregunta: “¿qué están haciendo para seguir embarazadas?, ¿qué tienen que hacer cuando el parto empieza? Nada, porque la fisiología ocurre espontáneamente", respondió. En esa línea, es usual que se destaque la asociación del parto con otro proceso fisiológico como el defecar. La idea que se resalta es que estos procesos no dependen de la voluntad de la persona sino que se dan de forma independiente y asociada a necesidades propias del organismo.

Sin duda, la idea de "espontaneidad" es clave en la explicación de la fisiología tanto en el parto como la crianza. Se apela a la espontaneidad para explicar todos y cada uno de los hitos fisiológicos del embarazo, el parto, nacimiento 
y crianza. Sin embargo, se trata de una espontaneidad que se produce en determinado contexto; es decir que, si bien los mecanismos fisiológicos ocurren como parte de nuestro funcionamiento como especie humana, necesitan también ciertas condiciones socioambientales y emocionales que no obstaculicen su emergencia y desarrollo.

La intimidad, la tranquilidad y la soledad son condiciones indispensables para que los procesos fisiológicos se produzcan sin dificultades; condiciones que distan de ser las habituales en las instituciones de salud donde se producen la mayoría de los partos y nacimientos. Es por ello que en los espacios de difusión de la fisiología del parto se transmite información acerca de estrategias para minimizar situaciones institucionales de adversidad. Por ejemplo, en una de las reuniones de Gestar, la coordinadora aconsejaba la utilidad de quedarse en el baño de la clínica durante el transcurso del trabajo de parto para evitar intromisiones. También les advertía a las parejas acerca de llevar a las clínicas sus propias pelotas, aceites esenciales o banquito de parto, en el caso que aquellas no contasen con ellos. La idea de personalizar el espacio va en línea con la tranquilidad necesaria para que se produzca el parto fisiológico.

Con la misma intención, en las charlas y talleres se recomienda realizar la mayor parte del trabajo de parto en la casa y trasladarse cuando las contracciones apuren su frecuencia, para que el contexto institucional no perjudique el proceso. En este sentido, la figura del parto planificado en el domicilio para el caso de las gestaciones saludables es planteada como la mejor opción para evitar esas interrupciones y cambios de contexto. Esta visión de la "espontaneidad en contexto" es clave en los relatos de las mujeres que suelen mencionar cómo el trabajo de parto se enlenteció en el momento de trasladarse a la institución.

Asumir que la fisiología es espontánea implica aceptar la importancia de la confianza como actitud ontológica frente a la ocurrencia del parto. Confianza en el cuerpo, y los procesos corporales propios de la especie humana y en la creencia que la intervención puede dañarlos es un eje central de esta perspectiva. Sin embargo, dicha confianza no se contrapone a estar atento a las condiciones ambientales que favorecen o entorpecen la fisiología, por el contrario, requiere de una atención cuidadosa del contexto en que se manifiesta. La "espontaneidad" se articula con los tiempos en los que se ocurre el trabajo de parto que son variables, inciertos e inesperados. Ello se opone a la lógica de estandarización que establece el sistema médico. "El enemigo de la fisiología es el tiempo", me repetía incansablemente una de las informantes, activista por los derechos reproductivos y sexuales de las mujeres en la escena del parto. La perspectiva biomédica clásica no solo no respeta las evidencias científicas y recomendaciones internacionales que respaldan el parto y el nacimiento fisiológico sino que tampoco observa "el bienestar materno fetal":

Yo creo que justamente el problema también con entender la fisiología es que el modelo dominante trabaja en función del tiempo, o sea, su criterio está basado en el tiempo, tantas horas para dilatar, si no dilataste en tantas horas hay algo mal, y no en el bienestar materno-fetal. Y un tiempo determinado, y si no sucede en los tiempos... pero la fisiología no tiene tiempos, porque escapa a esa medición, tiene que ver con otras cosas, entonces claro, como estamos acostumbradas a que se nos mida en función del tiempo... Entender que no, que esto no va de tiempo sino del bienestar materno fetal, si tú estás bien, si el bebé está bien, seguimos adelante, y bien también significa bien físicamente pero también bien emocionalmente y bien psicológicamente. (Entrevista con Lucía, activista por los derechos de las mujeres en el parto y madre de dos niñas, julio de 2018) 
El "bienestar materno-fetal" se garantiza, en parte, mediante el cuidado de las condiciones de parto descritas con anterioridad: la espontaneidad, la intimidad, los tiempos de cada díada madre-bebé, todas condiciones que presuponen el desarrollo de un parto fisiológico y que propician una conexión con las propias necesidades del cuerpo y una escucha de estas. Así es que "escuchar al cuerpo" se convierte en un elemento fundamental de la retórica fisiológica. Se les propone a las mujeres "conectarse" con el proceso de gestación, estar atentas a los cambios físicos y emocionales, e intervenir sobre ellos. Por ejemplo, desde un masaje en el perineo que prepara el piso pélvico para adquirir flexibilidad en el parto hasta una decodificación sensorial y emocional de la función de las contracciones y el dolor del trabajo de parto.

Los ejercicios de respiración, yoga prenatal, visualizaciones y meditaciones para conectar con el útero, el bebé y la experiencia propia del nacimiento y la alimentación saludable son elementos fundantes de esta educación corporal inherente a la retórica fisiológica que se difunde mediante los talleres, charlas, y "círculos de mujeres gestantes". Visibilizar y dar sentido a esa conexión es el puente para la construcción de una gramática del instinto, de la sabiduría del cuerpo que rige tanto la gestación y el parto como el vínculo que se establece después con los recién nacidos, en particular a través de la lactancia.

Las coordinadoras de los espacios de difusión de la fisiología suelen contar sus propias experiencias de parto y los aprendizajes a los que estas las llevaron respecto de sus propias corporalidades, es así que "escuchar al cuerpo" es un elemento operativo de este enfoque. En esa línea, Juana, la coordinadora de Gestar, revela una lección aprendida en su parto en casa. A nivel global, la experiencia para ella fue la de un parto respetado y fisiológico, sin embargo, le quedó cierta disconformidad por la insistencia de la partera de que pujara en la etapa final del expulsivo. Juana se sintió incómoda con la presión de la partera que no resonó con sus propias necesidades y su "escucha del cuerpo" y cuya consecuencia fue un pequeño desgarro. La anécdota destaca la centralidad de escuchar las indicaciones del propio cuerpo: guía soberana del desarrollo del trabajo de parto. Las mujeres que compartieron sus relatos durante esta investigación destacaron el valor de esas experiencias guiadas por sus sensaciones corporales e intuiciones. Las palabras de Noelia, quien, luego de un parto extremadamente intervenido alcanzó una experiencia fisiológica con su segunda hija, ejemplifican estas cuestiones:

Fui sintiendo la progresión de la intensidad de ese dolor, y fue genial porque... para mí fue genial sentir eso porque fue natural, fue totalmente fisiológico en el sentido de que fue al tiempo nuestro, fue al tiempo mío, al tiempo de mi bebé. (Entrevista con Noelia, madre de dos niños, mayo de 2018)

Asimismo, a las explicaciones acerca del valor y las condiciones de la fisiología del parir y nacer se suma la información de las etapas del parto y cómo intervienen las hormonas en cada una de ellas. En otro encuentro, Juana, explicaba las primeras instancias del trabajo de parto:

Cuando el parto detona, el bebé manda la primera señal de oxitocina al cerebro de la mujer. La oxitocina es la hormona del amor, está presente en todas las situaciones amorosas, es responsable de la eyección en el parto, la leche materna, el semen. Si hay adrenalina, la oxitocina se retira, es una hormona tímida. La oxitocina tiene una función mecánica, dado que es responsable de las contracciones pulsátiles. Y tiene también una función emocional que es un efecto de calma y amor. La oxitocina sintética, por su parte, anula la producción de oxitocina natural y no tiene la función 
de calma y amor. Si todos (dirigiéndose a los futuros padres) protegen la oxitocina, entonces van a ser buenos compañeros de parto. (Nota de campo del segundo encuentro de Gestar, febrero de 2018)

En estas palabras se observan elementos clave para comprender la función de las hormonas: se presenta la oxitocina, que es la hormona principal del parto, a la que se hace mención en muchísimas otras ocasiones, tal como documenté durante mi trabajo de campo. ${ }^{7}$ La regulación de la oxitocina se articula a la presencia de los compañeros, futuros padres. Más que espectadores de una escena femenina, se transforman en actores fundamentales del parto de sus hijas/os. ${ }^{8}$

Las explicaciones acerca del funcionamiento de las hormonas distinguen la oxitocina sintética de la natural; allí nuevamente se valoriza la fisiología: el mensaje principal sugiere que lo artificial no puede emular las virtudes de la naturaleza. Más aún, en el contexto hipermedicalizado de atención, en el que la aplicación de suero con oxitocina sintética es un procedimiento de rutina y es visto como el inicio de las actuaciones médicas que interrumpen, entorpecen y paralizan el trabajo de parto, siendo responsables en buena parte de los casos del requerimiento de las cesáreas (lo que los profesionales que alertan sobre estas cuestiones llaman "la cascada de intervenciones").

Vital en este proceso es la adrenalina, la hormona contraria a la oxitocina. Su aparición en la etapa de trabajo de parto activa es descripta como un entorpecedor significativo. Una de mis informantes me contó de la importancia de "no generar adrenalina en el rol de doula":

Vos estás douleando a alguien que le pasa algo parecido podés reaccionar y asustarte... o sea, vos tenés que liberar oxitocina completamente, y si empezás a liberar adrenalina, vos contagiás a la mujer de esa adrenalina; en el parto es lo peor que le podés hacer, entonces vos tenés que mantenerte en calma y en paz todo el proceso de la mujer; si acompañás en el parto, tenés que estar ahí pilas y con tus hormonas positivas, en el momento que tenés adrenalina vos empezás a contagiar el entorno. (entrevista a Martina, mamá de una niña, doula asesora en crianza, julio de 2018)

La tercera hormona que cobra relevancia en la escena del parto es la noradrenalina. Claudia, otra de las coordinadoras de Gestar, lo explica a las parejas gestantes:

En la siguiente fase, la expulsiva, el conjunto de hormonas cambia y aparece la noradrenalina, que dota a la mujer de una fuerza animal que le da la energía para empujar. No se crean esta frase de cuando venga el expulsivo no vas a tener fuerza porque es una fase placentera. Acá los compañeros pueden recordarles a las mujeres que les falta poco, que ya van a ver a sus bebés. (nota de campo segundo encuentro de Gestar, febrero de 2018)

Nuevamente la confianza en el cuerpo surge como dato central, esta vez mediada por la función de las parejas que en el momento necesario alcen su voz para restablecer la confianza en esa situación de vulnerabilidad y cansancio que supone el final del parto. En los relatos de partos documentados en esta investigación es frecuente que las parteras u obstetras que acompañan a las mujeres deslicen comentarios del tipo "si estás tan cansada ahora no vas a poder", con lo cual merma, en consecuencia, la concreta posibilidad de que lo logren. Por ello, las alertas y estrategias de acompañamiento sugeridas en
7. La oxitocina se articula con las explicaciones del amamantamiento y los efectos placenteros, de regulación emocional y física para la díada mamá-bebé, como también a otras experiencias de contacto piel con piel. Recuerdo cómo en una feria de crianza respetuosa una asesora en porteo (es decir, quienes ayudan a los adultos a portear, cargar en brazos, de forma adecuada, segura y saludable con elementos correctos como mochilas y fulares) contaba que gracias a esta práctica los bebés producían oxitocina de forma continua. El porteo se condice con la mirada fisiológica en tanto se apoya en la importancia del contacto piel con piel continuado, en especial durante los primeros nueve meses de vida, que se concibe como el período de exterogestación para un adecuado desarrollo emocional y físico.

8. En los encuentros que presencié, las mujeres gestantes asistieron acompañadas de sus parejas hombres. La interpelación a los padres en estos casos no obedece a un sesgo heteronormativo de estos dispositivos sino a las características del público asistente. 
los espacios de difusión son vitales instrumentos de empoderamiento para las mujeres que deciden protagonizar sus gestaciones.

Entender el funcionamiento hormonal implica respetar los contextos y escenas del parto e ilumina cómo la fisiología depende de una experiencia social concreta que va a facilitar u obstaculizar su desarrollo. En ese sentido, ese conocimiento formal de la naturaleza tiene la pretensión de transformarse en un lenguaje interpretativo de la experiencia corporal, herramienta vital para atravesar las etapas de la gestación al mismo tiempo que constituye información privilegiada que las mujeres movilizan en sus interacciones con el entorno médico a los fines de hacer valer sus derechos y deseos de parir:

\begin{abstract}
Empiezo a pujar. Entra una partera y me pone suero. "Por si perdés mucha sangre." Prepara algo y empieza a ponérselo al suero. ¿Qué estás poniéndome? Oxitocina, mami. ¿Qué?, ¡Sacame eso ya! No ves que ya estoy pujando. Tengo dilatación casi completa, ¿a vos te parece que necesito oxitocina sintética? Me puso cara de culo, me la sacó y me dijo "bueno, si te gusta sufrir vas a estar un buen rato acá", y se fue. (relato publicado en las redes sociales, grupo de información sobre experiencias de partos, mayo de 2018)
\end{abstract}

La retórica fisiológica organiza, fundamenta y visibiliza la función de los mecanismos hormonales en el parto y de los factores ambientales que pueden perjudicarla. Las explicaciones acerca del funcionamiento hormonal que brindan quienes difunden las bondades de la fisiología en el parto, tienen la cualidad de presentar a las hormonas no como meras sustancias producidas por un organismo que actúan dentro de él sino como mediadores, es decir, como sustancias que impactan en el entorno y en otras personas. Pues su efecto instaura modificaciones en el espacio relacional que rodea al parto: los compañeros, la propia mujer pariendo, el equipo de salud; todos se convierten en portadores y transmisores de las hormonas que circulan en ese entramado relacional y que lo dotan de sentido. Entender qué hormonas actúan, cómo lo hacen, qué significado tiene el dolor de una contracción y cómo el ambiente en el que transcurre el trabajo de parto es vital para su fluidez es un potencial fundamento a los fines de reposicionar a la mujer en el centro de la escena. En ese sentido, entender la dinámica hormonal que acompaña las distintas fases se torna en un aliado de la confianza de las mujeres en sus cuerpos a la hora de parir.

A continuación, analizo los cruces entre maternidad, naturaleza y cultura, y planteo que la retórica fisiológica favorece prácticas de empoderamiento de la mujeres a partir de generarles confianza en su capacidad de parir y seguridad en los procesos naturales y espontáneos de sus cuerpos y el de sus hijos/as.

\title{
Reinventando los sentidos de la naturaleza o nuevos sentidos culturales para la maternidad
}

El lenguaje de la fisiología ilumina la experiencia del parir y criar a partir de dotarla de una racionalidad biológica que decodifica los procesos corporales de las mujeres y de las niñas y los niños y los traduce a una información asequible por las mujeres que les permite esperar, sentir y articular condiciones de respeto de sus cuerpos y el de sus hijos e hijas. En las experiencias de las mujeres entrevistadas, y con las que conversé informalmente en los grupos y talleres, la información al respecto es la puerta de entrada a una nueva forma de entender y vivir la maternidad. 
Algunas mujeres, tras largas noches de desvelos, acceden a información sobre los ritmos del sueño infantil; otras, motivadas por la cantidad de veces que sus hijos les reclaman la teta se tranquilizan cuando conocen la fisiología del amamantamiento; y en otras, las necesidades de sus bebés de estar en brazos de forma casi constante las llevan a encontrar información sobre el contacto piel con piel y la crianza en brazos.

En cualquier caso, el conocimiento de la fisiología se convierte en un momento bisagra en sus trayectorias como madres que buscan una conexión diferente con sus hijos y como mujeres que buscan un mayor respeto por la naturaleza de sus cuerpos.

Los espacios de circulación de la información, que describimos aquí, las convocan a romper con los conocimientos, consejos y recomendaciones que usualmente reciben del sistema de salud respecto de la gestación, el parto y la crianza. Este hallazgo coincide con el trabajo de Happel-Parkins y Azim (2016), que destaca el valor de los conocimientos que tienen las mujeres como una variable central a la hora de cuestionar la autoridad médica del parto. En este sentido, el respeto por la fisiología como base de la atención médica del parto se opone al modelo de atención tecnocrático descripto por el ya clásico estudio de la antropóloga Davis-Floyd (1992, 2004), caracterizado por la hipermedicalización del parto y el nacimiento. La virtud del análisis de Floyd (2003) es que ofrece una perspectiva culturalista respecto del parto y el nacimiento y muestra que el modelo tecnocrático no es solo un modelo de atención de salud, sino el reflejo de un sistema cultural en el cual a las mujeres se les enseña la superioridad de la medicalización por encima de su propia capacidad de gestar y dar a luz. En este sentido, son las mismas mujeres quienes colaboran con el sostenimiento del sistema al contribuir a su legitimidad con su propia adhesión.

Teniendo en cuenta estas premisas, sugiero que la retórica fisiológica se torna en una herramienta contracultural, en tanto disputa tanto el modelo médico hegemónico en la atención del embarazo y el parto como el control pediátrico y las recomendaciones psicológicas y pediátricas en la crianza. Bajo esta óptica, la naturaleza emerge como un tipo de conocimiento liberador que coloca la agencia de las mujeres y los niños, y la autonomía de sus decisiones, en primer lugar. Comprender el carácter liberador que puede causar esta retórica implica tener en cuenta el contexto en el que este discurso cobra sentido. Es decir, la liberación de la opresión medicalizadora del parto y de la influencia de las recomendaciones pediátricas clásicas que colocaron la crianza por fuera del dominio del propio saber materno y de las necesidades de las niñas y niños. Sin duda que bajo este prisma emergen otros saberes que acompañan y a veces juzgan duramente las experiencias de las mujeres. En efecto, la documentación de las discusiones y comentarios que se dan en las redes sociales y algunas experiencias que cuentan mis entrevistadas dan cuenta de la emergencia de nuevas normatividades que pueden asociarse a culpabilidades y desconciertos en las trayectorias de las mujeres madres. ${ }^{9}$ Sin embargo, el empoderamiento, como efecto, es una dimensión central a tener en cuenta en los análisis de las experiencias concretas. Las mujeres, bajo la retórica fisiológica son impulsadas a confiar en sus cuerpos para tomar decisiones de crianza que otrora estarían guiadas por la expertise ajena (pediátrica, obstétrica o de familiares). La apelación a conectar con la naturaleza instintiva tiene por efecto potenciar la agencia y el reconocimiento del valor de sus propias emociones y las de sus hijos como rectoras de la experiencia de maternar.
9. Las experiencias concretas de empoderamiento en tensión con la construcción de nuevas normatividades e ideales de crianza que podrían generar efectos culpabilizadores, así como los modos en que las mujeres en mi trabajo de campo construyen sororidades que redefinen los mandatos y habilitan las ambigüedades entre los discursos y las prácticas ameritan ser abordadas en un artículo en sí mismo. 
10. Sería interesante indagar cómo se articula la retórica fisiológica del parto y la crianza en las nuevas configuraciones familiares que no son heteronormativas ni tampoco responden necesariamente a

arreglos conyugales sino que, en algunos casos, se inscriben en acuerdos de coparentalidad, o maternidades/paternidades solas por elección.
Por otro lado, la retórica fisiológica es contracultural en tanto se opone a las ideas acerca de la experiencia de la maternidad como mandato o esclavitud, y a los modelos tradicionales de crianza, organización del espacio doméstico y regulación de las relaciones de autoridad entre progenitores y niños. Aquí los hombres y padres adquieren protagonismo desde el parto sosteniendo y cuidando la díada mamá-bebé los primeros meses, al tiempo que sus emociones y necesidades de apego cobran sentido en este modelo de paternar. ${ }^{10} \mathrm{En}$ base a la interpretación de sus hallazgos, Bobel (2002) afirma que las "madres naturales" podrán resistir algunas restricciones capitalistas y tecnológicas para la vida familiar pero no resisten las concepciones esencializadas y romantizadas de la mujer en una división del trabajo sexual clásica. Por el contrario, durante mi trabajo de campo, detecté tanto la asistencia activa de varones a los talleres de preparación para el parto como sus genuinos intentos de expresar sus emociones en el proceso de devenir padres. "Yo me di cuenta con el tiempo que yo la empujé a que acepte la cesárea, yo ahora no quiero que me pase eso, quiero estar preparado... sentí que la dejé sola", reflexionaba emocionado un padre en un taller de información para parir después de cesárea con relación al parto de su primera hija. También me encontré con relatos de mujeres que dan cuenta de que sus compañeros son partenaires en pie de igualdad en lo que respecta a las tareas de cuidado familiar. Dar la teta, más que en una forma de esclavitud se convierte, para muchas familias, en una invitación a repensar y producir las condiciones necesarias para que esas lactancias sean exitosas. Sin duda que esas condiciones incluyen la presencia en el espacio doméstico de los hombres, mediante el cuidado de la mujer que está amamantando y la organización de los quehaceres domésticos. Esto supone tanto un cuestionamiento social al mercado de trabajo como una reacomodación familiar a los requerimientos de cuidado mencionados.

Por otro lado, una cuestión central que atraviesa la crítica sobre las "maternidades naturales" es la visión que esta perspectiva asume respecto del lugar y el sentido de la naturaleza. Para Bobel (2002, p. 126), en este modelo, las mujeres conciben a la naturaleza como la guía para el comportamiento humano; en palabras de la autora, están "forzadas por la naturaleza" (forced by nature), porque en última instancia la ubican en el lugar de autoridad suprema. En ese sentido, Bobel compara la sumisión a la naturaleza de este enfoque con el control de las mujeres por parte de los hombres o de la religión. Para la autora, esta visión es problemática en tanto niega las múltiples maneras en que la noción de naturaleza es en realidad una construcción cultural e interpretativa al tiempo que subordina a las mujeres a un nuevo mandato. Con un espíritu similar, el trabajo de Lázzaro (2017) también subraya los efectos esencializantes que se deducen de la concepción de los cuerpos femeninos en carácter de mamíferos y portadores de un instinto universal presocial.

No obstante, y en consonancia con los hallazgos de esta investigación, considero que el valor de este tipo de mirada de la naturaleza/fisiología como guía para la acción social en el proceso de parir y criar radica en interpelar los modelos sociales clásicos de entendimiento del vínculo naturaleza-cultura que reposiciona a la fisiología del lado de la transformación social y a la cultura, del lado del conservadurismo/naturalismo.

La atención hipermedicalizada del parto y el modelo tradicional de crianza se contraponen con el enfoque fisiológico del parto y la crianza. Reponer los sentidos y procesos de significación social es vital para entender estas nuevas asociaciones entre naturaleza y cultura. En esta línea, este artículo dialoga con los trabajos que documentan los sentidos de la naturaleza en el parto desde 
la perspectiva de las propias mujeres que interpretan y utilizan en sentido liberador la noción de naturaleza (Klassen, 2001; Tornquist, 2002, 2004; Martin, 2006; Abdala, 2016, entre otros).

Estas mujeres, en palabras de Klassen (2001), están creando nuevas visiones del nacimiento (y de la crianza, agregaría) que colocan tanto a ellas como a sus niños y niñas en el centro de la escena. Tal como insiste una de mis informantes -feminista y activista en estas temáticas-, "están cambiando el relato cultural del parto", es decir, el relato del parto como evento médico, riesgoso y urgente. Interrogar la noción de naturaleza implica desentrañar el conjunto de interpretaciones que se hacen sobre el parto fisiológico, los contextos específicos que permiten u obstaculizan su ocurrencia y los actores sociales que le dan sentido, lo que evidencia así el carácter social de lo natural (Dutra y Meyer, 2007; Logsdon y Smith Morris, 2017). Acuerdo con Mansfield (2008) respecto a que ello desafía el pensamiento tradicional académico de las ciencias sociales que caracteriza a los enfoques de maternidad y crianza natural como esencialistas y en sintonía con representaciones de género tradicionales y conservadoras. Lo "natural" aquí se vincula con lo saludable y genuino, el placer y la autonomía femenina; por el contrario, lo "cultural", del lado de la intervención médica innecesaria, la ausencia de salud y el autoritarismo médico. $Y$ es precisamente en esta reconversión de significados de la naturaleza/fisiología donde radica la potencialidad transformativa del movimiento de parto y crianza natural. La retórica fisiológica no constriñe a las mujeres al histórico mandato natural de la maternidad tan bien analizado por los estudios feministas clásicos. Por el contrario, la fisiología refuerza la capacidad de agencia de aquellas mujeres que eligen ser madres y encuentran en la naturaleza una clave interpretativa de la maternidad y la crianza. En estos casos, la retórica fisiológica se convierte en una herramienta vital de circulación de sus voces en diversos espacios sociales (tanto doméstico y familiar como médico y público). En palabras de Fornes:

Aunque se apoyen discursivamente en el instinto maternal, la recuperación de la intimidad y la experimentación de tiempos y procesos fisiológicos, la serie de toma de decisiones y experiencias vividas exceden la apelación a lo natural y las decisiones privadas, colocando a la experiencia de parto en la arena política misma. (Fornes, 2011, p. 148)

\section{Consideraciones finales}

En este artículo analicé desde una perspectiva sociológica cómo los discursos de parto fisiológico y crianza fisiológica conciben y dan sentido a la noción de "naturaleza". En este contexto se entiende a la naturaleza bajo el concepto de fisiología, es decir, los procesos corporales que ocurren de forma espontánea porque forman parte de nuestra existencia como especie humana. El parto y algunos hitos del desarrollo infantil se conciben dentro de este marco y ello implica entender una serie de procesos y prácticas como el amamantamiento, el colecho, el control de esfínteres, la motricidad, entre otras, bajo la pauta del respeto de las necesidades y ritmos de los cuerpos, ya sea de las mujeres pariendo o de los niños y las niñas creciendo.

En primer lugar, me detuve en una descripción general que engloba al parto y a la crianza y que busca situar las coordenadas de la naturaleza en un continuum de experiencias sociales que comienza en el nacimiento. Luego, focalicé en la descripción y análisis de la fisiología del parto y lo que denominé como la "retórica fisiológica del parto" para englobar los modos de argumentación 
y circulación de este enfoque en lo que refiere a las capacidades innatas de las mujeres en el proceso de dar a luz.

Esta descripción me permitió observar los efectos empoderantes que la retórica fisiológica tiene en las vitales experiencias de parir y criar. La naturaleza aquí, lejos de ser un discurso a los fines de opacar y /o legitimar las relaciones sociales (dinámicas desiguales y conservadoras de roles de género) que estructuran la experiencia de la maternidad, se convierte en un saber que alienta a las mujeres, les genera confianza y transforma una escena gobernada por el discurso médico hegemónico y una narrativa patriarcal del parto y la crianza.

Los significados de la noción de naturaleza que se conjugan en la perspectiva fisiológica del parto y la crianza podrían pensarse en el marco de la problematización sobre los binarismos naturaleza-cultura que viene dándose en el campo de la antropología y los estudios feministas en las últimas décadas (Ortner, 1974; Cheah, 1996; Kerin, 1999; Franklin, 2003, entre otros). Estudios socioantropológicos basados en diferentes referentes empíricos y diversas áreas temáticas reflexionan acerca de la relación entre la noción de naturaleza y la de cultura, ya sea postulando el fin de dichos binarismos o su reconfiguración a partir de nuevas significaciones que son movilizadas en contextos sociales específicos.

Por ejemplo, en el campo de las tecnologías de reproducción asistida y el desarrollo de la nueva genética se plantea una visión de la naturaleza ya no como algo dado, sino como pasible de ser transformada por las intervenciones tecnológicas y por el impacto del contexto socioambiental. La posibilidad concreta que inaugura la nueva ingeniería genética de transformar la naturaleza impulsa múltiples debates. Algunos autores plantean que las diferencias entre la naturaleza y la cultura han colapsado. Es el caso de Donna Haraway (1991), quien utiliza la metáfora de la implosión para describir cómo la naturaleza y la cultura han sido interpenetradas una con la otra. En contraste, Paul Rabinow (1996) plantea que el dominio de la naturaleza o la biología no puede ser visto como una condición a priori para lo social porque lo social (la cultura) es el modelo para una nueva naturaleza redefinida a través de la tecnología. La naturaleza, entonces, se moldea a partir de la cultura al tiempo que la cultura se naturaliza, en tanto se inscribe en la misma biología, lo que da por resultado una suerte de inversión de los modos clásicos de vinculación entre la naturaleza y la cultura. En esa línea, Marilyn Strathern (1992) analiza cómo la naturaleza está siendo "asistida" por la tecnología, por lo que no es posible continuar dándole un estatuto ontológico superior. Por el contrario, Illina Singh (2012) plantea que la década del cerebro y el proyecto de genoma humano -lejos de volver fútil la relación naturaleza-cultura- vuelven a insistir en su importancia. Para la autora, a partir del desarrollo de la genética y la consecuente visualización de las dimensiones moleculares de la vida, la división entre "herencia y adquisición" se convirtió materialmente más real.

Otras autoras recuperan esta idea acerca de cómo el par "naturaleza-cultura" es puesto a generar nuevas significaciones enmarcadas en contextos sociales específicos que potencian las diferencias entre uno y otro (Franklin, Lury y Stacey, 2000). Así es que, al tiempo que algunos autores y autoras analizan la disolución de los binarismos naturaleza-cultura, otras/os plantean su potencialidad analítica y vigencia en determinados espacios sociales.

La relevancia de este debate es visibilizar cómo la relación naturaleza-cultura se torna un vínculo potente de significación social en algunos contextos y pierde 
efectividad en otros (siempre dependiendo de quiénes sean los actores que definan la validez de estas nociones). Asimismo, estos debates nos enseñan acerca de las múltiples nociones de naturaleza y de cultura que están en juego y cómo se trata de definiciones situadas (en los autores citados surgen en el campo de nuevas biotecnologías médicas como lo son la ingeniería genética y la reproducción asistida). Sin duda, la perspectiva de Raymond Williams (1980) respecto de cómo la naturaleza es un concepto polisémico adquiere renovada vigencia.

En esa línea, al analizar la naturaleza en clave de fisiología como una noción susceptible de generar efectos empoderantes en las mujeres, nos colocamos en oposición a la definición de naturaleza propia de la biomedicina, que visualiza a los procesos fisiológicos del parto y nacimiento como eventos de riesgo y de forzosa intervención médica. Es en ese marco situado y específico que cobra dimensionalidad la noción de naturaleza y adquiere sentido contracultural la fisiología del parto y la crianza.

\section{Sobre la autora}

María Jimena Mantilla es doctora en Ciencias Sociales por la Universidad de Buenos Aires y actualmente se desempeña como investigadora adjunta del Consejo Nacional de Investigaciones Científicas y Técnicas (CONICET), con sede en el Instituto de Investigaciones Gino Germani.

\section{Financiamiento:}

La investigación que da pie al artículo no cuenta con financiamiento específico. Se inscribe en el marco de mis tareas como investigadora del Consejo Nacional de Investigaciones Científicas y Técnicas.

\section{Agradecimientos:}

Agradezco a las mujeres entrevistadas y a los y las participantes de los espacios de difusión de la perspectiva fisiológica que compartieron conmigo sus vivencias, dudas y temores sobre la maternidad y la crianza. 


\section{Q Referencias bibliográficas}

" Abdala, L. (2016). Exploración sociológica de los significados y prácticas de la maternidad en mujeres que reivindican "partos humanizados" y "rrianzas naturales" (Tesis de licenciatura). Facultad de Humanidades y Ciencias, Universidad Nacional del Litoral, Paraná, Argentina.

» Badinter, E. (1981). ¿Existe el amor maternal? Barcelona: Paidós.

»Badinter, E. (2010). La mujer y la madre. Madrid: La esfera libros.

» Bobel, C. (2002). The Paradox of Natural Mothering. Filadelfia: The Temple University.

»Carli, S. (Comp.) (1999). De la familia a la escuela. Infancia, socialización y subjetividad. Buenos Aires: Santillana.

»Castrillo, B. (2014). La perspectiva de género en el estudio de la intervención médica en el parto. I Congreso de Investigación Cualitativa en Ciencias Sociales. University of Illinois y Universidad Siglo 21, Córdoba, Argentina.

"Cheah. P. (1996) Mattering. Bodies that matter: on the discursive limits of sex by Judith Butler, Volatile Bodies: Toward a Corporeal Feminism by Elizabeth Grosz. Diacritics, 26, (1) 108-139

" Cosse, I. (2009). La emergencia de un nuevo modelo de paternidad en Argentina (19501975). Estudios demográficos y urbanos, 71, 429-462.

" Cosse, I. (2010a). Argentine mothers and fathers and the new psychological paradigm of child- rearing (1958-1973). Journal of Family History, 35(2), 180-202.

"Cosse, I. (2010b). Pareja, sexualidad, familia en los años sesenta. Buenos Aires: Siglo XXI.

"Davis-Floyd (1992). Birth as an American Rite of Passage. Berkeley: University of California Press.

»Davis-Floyd, R. (2003). The technocratic model of birth. En S. Tower Hollis, L. Pershing y M. Jane Young (Eds.), Feminist Theory in the Study of Folklore. Illinois: University of Illinois Press, 297-326

"Del Olmo García, C. (2013). ¿Dónde está mi tribu? Maternidad y crianza en unas sociedad individualizada. Madrid: Clave Intelectual.

»Dutra, I. y Meyer, D. (2007). Parto natural, normal e humanizado: termos polisémicos. Revista Gaucha de Enfermagem, 28(2), 215-22.

" Faircloth, C. (2013). Militant lactivism? Attachment parenting and intensive motherhood in the UK and France. Oxford: Berghahn Books.

» Faur, E. (2014). El cuidado infantil en el Siglo XXI. Mujeres malabaristas en una sociedad desigual. Buenos Aires: Siglo XXI.

" Floyd, D. (2004). Birth as an American rite of passage. Berkeley: University of California Press.

» Fedele, A. (2016). "Holistic mothers" or "Bad mothers". Challenging biomedicals models of the body in Portugal. Religion and Gender, 6(1), 95-111.

» Felitti, K. (2012). Nuevas formas de atención del parto en la argentina en los años sesenta y setenta: debates y experiencias. IX Congreso Iberoamericano Ciencia Tecnología y Género, Universidad de Sevilla, Sevilla, España. 
" Fornes, V. (2011). Parirás con poder... (pero en tu casa). El parto domiciliario como experiencia política contemporánea. En K. Felitti (Coord.), Madre no hay una sola. Experiencias de maternidad en la Argentina (pp. 133-154). Buenos Aires: Ciccus.

" Franklin, S. (2003). Re-thinking nature-culture. Anthropology and the new genetics. Anthropological Theory, 3(1), 65-85.

» Franklin, S., Lury, C. y Stacey, J. (2000). Global nature, Global culture. Londres: Sage.

" Happel-Parkins, A. y Azim, K. (2016). At pains to consent. A narrative inquiry into women's attempts of natural childbirth. Women and Birth, 29(4), 310-320.

" Haraway, D. (1991). Simians Cyborgs and Women: the re invention of nature. Nueva York: Routledge.

"Jerez, C. (2015). Partos humanizados, clase y género en la crítica a la violencia a las mujeres en los partos (Tesis de licenciatura). Facultad de Filosofía y Letras, Universidad de Buenos Aires, Buenos Aires, Argentina.

" Kerin, J. (1999). The matter at hand: Butler, Ontology and the Natural Sciences. Australian Feminist Studies, 14, (29),91-104

"Klassen, P. (2001). Sacred maternities and postbiomedical bodies: religion and nature contemporary homebirth. Signs, 26(3), 775-809.

"Lázarro, A. (2017). Cuerpos al natural: la construcción de la naturaleza y sus tensiones en el movimiento de Parto Humanizado. Pilquen, 20(3), 82-94.

"Liedloff, J. (2011). El concepto del continuum: en busca del bienestar perdido. Tenerife: Obstare.

"Logsdon, K. y Smith Morris, C. (2017). An ethnography on perceptions of pain in Dutch natural childbirth. Midwifery, 55, 67-74.

"Mansfield, B. (2008). The social nature of natural childbirth. Social Science $\&$ Medicine, 66(5), 1084-1094.

» Mantilla, M. J. (2019). “Cuerpos, niñez y crianza: cartografías corporales de la infancia en el modelo de crianza respetuosa en la Argentina". Revista Uruguaya de Antropología y Etnografía, 4(1), 61-75.

» Martin, E. (2006). A mulher no corpo. Uma análise cultural da reprodução. Río de Janeiro: Garamond.

" Martucci, J. (2015). Why breastfeeding? Natural motherhood in Post-War America. Journal of Women's History, 27(2), 110-133.

" Meruane, L. (2018). Contra los hijos. Santiago de Chile: Random House.

" Organización Mundial de la Salud (2015). Declaración de la OMS sobre tasas de cesárea. Ginebra, Suiza: Organización Mundial de la Salud.

》 Ortner, S. B. (1974). Is female to male as nature is to culture? En M. Z. Rosaldo y L. Lamphere (Eds.), Woman, culture, and society (pp. 68-87). Stanford: Stanford University Press.

"Sánchez de Bustamente, M. (2017). Mamis famosas. Narrativas biográficas de la maternidad en la cultura de masas (Tesis de maestría). Facultad de Ciencias Sociales, Universidad de Buenos Aires, Buenos Aires, Argentina.

"Schwarz, P. (2010). Construcción de la maternidad en el encuentro de la institución médico ginecológica con mujeres de clase media de la ciudad de Buenos Aires. Sexualidad, Salud y Sociedad - Revista Latinoamericana, 6, 83-110.

"Sears, W. y Sears, M. (1960). The attachment parenting book: A commonsense guide to understanding and nurturing your child. Boston: Little, Brown \& Company. 
" Singh, Illina. (2012). Human development, nature and nurture: working beyond the divide. Biosocieties, 7, 308-321.

" Strathern, Mary. (1992). After Nature: English Kinship in the Late Twentieth Century. Cambridge: Cambridge University Press.

»Tenenbaum, T. (2019). El fin del amor. Coger y querer en el siglo XXI. Buenos Aires: Ariel.

" Tornquist, S. (2002). Armadilhas da Nova Era: Natureza e Maternidade no ideário da humanização do parto. Estudos Feministas, 2, 483-492.

» Tornquist, S. (2004) Parto e poder. O movimento pela humanização do parto no Brasil. Tese

» Tese apresentada ao Programa de Pos-Graduação em Antropologia para obtenção do titulo de dotor em Antropologia

»Williams, R. (1980). Ideas of Nature. En Problems in Materialism and Culture. Londres: Verso.67-85 